\section{KN-14 Trapping a transient state in DNA mismatch repair: the MutS/MutL sliding clamp loads MutL on DNA}

Titia K. Sixma ${ }^{1}$

1. Division of Biochemistry Netherlands Cancer Institute, Amsterdam, Netherlands

email: t.sixma@nki.nl

Flora S. Groothuizen ${ }^{1}$, Ines Winkler ${ }^{2}$, Michele Cristóvão ${ }^{2}$, Alexander Fish ${ }^{1}$, Herrie H.K. Winterwerp ${ }^{1}$, Annet Reumer ${ }^{1}$, Andreas D. Marx ${ }^{2}$, Nicolaas Hermans, Robert A. Nicholls ${ }^{4}$, Garib N. Murshudov ${ }^{4}$, Joyce H.G. Lebbink $^{3}$, Peter Friedhoff ${ }^{2}$, Titia K. Sixma ${ }^{1}$

${ }^{1}$ Netherlands Cancer Institute (NKI), Amsterdam, the Netherlands, ${ }^{2}$ Justus-Liebig-University, Giessen, Germany, ${ }^{3}$ ErasmusMC, Rotterdam, the Netherlands, ${ }^{4} \mathrm{LMB}$, Cambridge, UK

To avoid mutations in the genome, DNA replication is followed by DNA mismatch repair (MMR). This process starts when a MutS homolog recognizes a mismatch and undergoes an ATP-dependent transformation to an elusive sliding clamp state. How this transient state promotes MutL homolog recruitment and activation of repair is unclear.

Here we present the crystal structure of the MutS/MutL complex where we trap this transient state, by making use of highly specific single-cysteine crosslinking. The resulting structures (4.7-7.6 A) have suprisingly large conformational changes that were validated by FRET, binding studies and mutagenesis and interpreted in terms of the MMR cycle.

The structure captures MutS in the sliding clamp conformation, where tilting of the MutS subunits across each other pushes DNA into a new channel, and reorientation of the connector domain creates an interface for MutL with both MutS subunits. Our work explains how the sliding clamp promotes loading of MutL onto DNA, to activate downstream effectors. We thus elucidate a crucial mechanism that ensures that MMR is initiated only after detection of a DNA mismatch.

Keywords: Low resolution refinement, crosslinking, DNA repair, FRET, SPR, MutS, MutL

\section{$\mathrm{KN}-15$ Molecular mechanisms of RNA polymerase I and III transcription \\ Christoph W. Müller ${ }^{1}$}

1. European Molecular Biology Laboratory (EMBL), Heidelberg, Germany

email: cmueller@embl.de

RNA polymerase (Pol) I and Pol III mainly synthesize the non-coding RNA components required for ribosome assembly and protein synthesis in eukaryotes. Pol I synthesizes precursor rRNA that is subsequently processed into $25 \mathrm{~S}, 18 \mathrm{~S}$ and $5.8 \mathrm{~S}$ rRNA, while Pol III produces small RNAs such as tRNA, 5S RNA and U6 snRNA. The Pol I and Pol III transcription initiation machineries are carefully regulated in healthy cells, while misregulation of Pol I and Pol III transcription is observed in a variety of cancers. Our group uses an integrated structural biology approach combining X-ray crystallography, single-particle electron microscopy and chemical crosslinking mass spectrometry to study the Pol I and Pol III enzymes and their transcription initiation complexes. We have determined the crystal structure of the 14-subunit RNA polymerase I from Saccharomyces cerevisiae at $3.0 \AA$ resolution. The Pol I structure shows a very wide DNA-binding cleft that is occupied by an extended loop mimicking DNA. The Pol I-specific subunits A12.2 and A49-A34.5 provide additional functionality to the enzyme. The crystal structure of Pol I will be compared to the cryo-electron microscopy structure of the 17-subunit Pol III enzyme. Recruitment of Pol III to tRNA-encoding genes requires the general transcription factors (TF) IIIB and IIIC. TFIIIC is a multi-subunit protein composed of two subcomplexes. We used chemical cross-linking mass spectrometry to determine the overall architecture of TFIIIC and to position crystal structures corresponding to about $2 / 3$ of the entire TFIIIC complex.

Keywords: RNA polymerases, transcription, multi-subunit complexes, integrated structural biology 